\title{
General Psychiatry Prevalence and dynamic features of psychological issues among Chinese healthcare workers during the COVID-19 pandemic: a systematic review and cumulative meta-analysis
}

\author{
Huijuan Zhang (10 , ${ }^{1}$ Wei Li, ${ }^{1}$ Hui Li, ${ }^{2}$ Caidi Zhang, ${ }^{1}$ Jinjing Luo, ${ }^{1}$ Yikang Zhu, ${ }^{1}$ \\ Hui Wu, ${ }^{3}$ Zhuoying Zhu, ${ }^{4,5}$ Yifeng Xu (D) ,1,4 Jijun Wang, ${ }^{1,4}$ Chunbo Li ${ }^{1,4}$
}

To cite: Zhang H, Li W, Li H, et al. Prevalence and dynamic features of psychological issues among Chinese healthcare workers during the COVID-19 pandemic: a systematic review and cumulative metaanalysis. General Psychiatry 2021;34:e100344. doi:10.1136/ gpsych-2020-100344

- Additional supplemental material is published online only. To view, please visit the journal online (http://dx.doi.org/10.1136/ gpsych-2020-100344).

Received 17 July 2020 Revised 01 February 2021 Accepted 21 March 2021

Check for updates

(C) Author(s) (or their employer(s)) 2021. Re-use permitted under CC BY-NC. No commercial re-use. See rights and permissions. Published by BMJ.

For numbered affiliations see end of article.

Correspondence to

Dr Hui Li; lihuindyxs@163.com

Dr Chunbo Li;

chunbo_li@163.com

\section{ABSTRACT}

Background The COVID-19 pandemic has placed tremendous pressure on healthcare workers who are at the frontline in the battle against it, causing various forms of psychological distress.

Aims To outline the prevalence and dynamic changing features of medical workers' psychological issues and to review the related national policies during the COVID-19 pandemic in China.

Methods We systematically searched for studies on the psychological states of Chinese medical staff from 1 January 2020 to 8 May 2020. The aggregate prevalence of each psychological issue was calculated and plotted to observe the changes over time. Policies on psychological support for medical staff during the pandemic were reviewed to explore their link to the prevalence of psychological problems.

Results In total, 26 studies were included in this study. A total of 22062 medical workers were surveyed from 31 January to 27 February 2020. Medical staff exhibited a substantial prevalence of anxiety symptoms, depression symptoms, stress-related symptoms and sleep problems during the survey period, with aggregate prevalence rates of $27.0 \%, 26.2 \%, 42.1 \%$ and $34.5 \%$, respectively. Cumulative meta-analyses revealed that the prevalence of psychological distress peaked at the beginning of the pandemic and subsequently exhibited a slow downward trend.

Conclusion Chinese medical staff displayed significant psychological disturbance during the outbreak of the COVID-19 pandemic, which was especially severe at the early stage. Implementing supportive policies may help alleviate ongoing psychological problems in healthcare workers.

\section{INTRODUCTION}

COVID-19 is an ongoing pandemic of SARS-CoV-2, ${ }^{2}$ which spread nationally over a short period. By 8 May, COVID-19 had spread to 215 countries or territories around the world, resulting in more than 3.7 million confirmed cases of infection and nearly
260000 deaths. $^{3}$ In China, the confirmed cases also amounted to over $82000 .{ }^{4}$ The National Health Commission of China classified COVID-19 as a class B infectious disease on 20 January and quickly issued a multitude of preventive measures and treatments for the disease. ${ }^{56}$

The arduous 'war' against COVID-19 has been inseparable from the efforts of medical health workers. Since the outbreak of the pandemic, a total of 344 national medical teams have been dispatched nationwide, and the total number of medical personnel exceeded 40000. Additionally, numerous healthcare workers have stayed in their local hospitals to battle the pandemic. In this situation, medical workers are under tremendous pressure. ${ }^{78}$ They are faced with a higher risk of infection and increased workload. The deterioration of the patients' physical and psychological health may cause exhaustion, even result in job burnout. ${ }^{9}$ Moreover, because of the increased workload, healthcare workers found it difficult to fulfil social roles such as looking after children or elderly family members. Studies have shown that workers feared the risk of transmitting the disease to family members or friends. Thus, they were often increasingly isolated over extended periods of time, which caused further aggravation of psychological conditions and stress.

Several cross-sectional studies have been designed to assess the mental status of healthcare workers in China during the pandemic, and the studies have found a higher prevalence of mental health and psychosocial problems among healthcare workers than in the general population..$^{10}$ Depression and anxiety are particularly prominent. ${ }^{11}$ Therefore, the psychological problems of medical 
workers have received attention at the policy level in China. ${ }^{12}$ From the early stage of the pandemic, a series of related national policies have been issued to promote the care of mental well-being among healthcare workers, such as ensuring adequate supplies of medical equipment, improving the work environment and increasing psychological support for frontline workers. ${ }^{13}{ }^{14}$ However, a previous study had found that the mental well-being of frontline medical workers was still impacted despite these measures. Indeed, data indicate that healthcare workers may have a higher prevalence of sustained symptoms of depression and anxiety even after the pandemic. ${ }^{15} 16$

To our knowledge, no systematic review on the changing features of frontline medical workers' psychological status in China during the COVID-19 pandemic has been conducted. In this study, we reviewed previous cross-sectional studies and the main public intervention policies aimed at supporting frontline workers' mental well-being. The aims of this article were as follows: (1) to describe the psychological status of medical workers at different time points during the COVID-19 pandemic in China, and (2) to preliminarily explore the impact of national policies on the psychological well-being of medical workers.

\section{METHODS}

\section{Source of data}

Prevalence of psychological distress among Chinese healthcare workers

Data on the psychological status of healthcare workers were retrieved from electronic databases. Two researchers (CZ and JL) systematically and independently searched the following electronic databases: PubMed, Embase, the Cochrane Library, E. B. Stephens Company database, Web of Science, ALOIS, PsycINFO, Cumulative Index to Nursing and Allied Health Literature database (CINAHL), ClinicalTrials.gov, the Chinese National Knowledge Infrastructure (CNKI), Sinomed, Wanfang Data and Chongqing VIP database. Included results were from the period of onset of COVID-19 to 8 May 2020. English keywords included 'novel coronavirus', '2019nCoV', 'COVID-19', '2019 n-COV', 'coronavirus disease', 'COVID-19', 'mental', 'psycho*', 'depress*', 'anxiety*', 'stress', 'trauma*', 'insomnia*', 'sleep', 'medical staff', 'healthcare worker*', 'doctor*' and 'nurse*'. Search words in Chinese were ‘新型冠状病毒肺炎', ‘新冠病毒', ‘新冠肺炎’, ‘2019冠状病毒’, ‘2019新型冠状病毒感染’, '2019-nCOV肺炎', ‘新型冠状病毒*', “心理*', ‘抑郁”, “焦

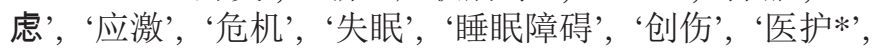
“医务*', ‘医生' and '护士’. We included cross-sectional studies that met the following criteria: (1) the research subjects were Chinese medical staff, such as doctors, nurses and technicians; (2) the research time was after the outbreak of the pandemic; and (3) studies included were those that measured the prevalence of a specific mental illness with validated rating instruments. Details of selected articles were collected using standardised data extraction templates, including general study information (trial name, author, publication date and study location), study characteristics (study design, assessment tools and survey time span), population characteristics (sample size, age, gender and occupation), results of psychological symptom scales, as well as information needed for assessment of risk of bias and evaluation of research quality. In the articles that provided survey time, we chose the date of the survey or the midpoint of survey time as the sampling time. For articles that did not provide survey time, we contacted the authors to obtain the information or chose the submission time as the sampling time. Two researchers (WL and HL) individually screened the articles and extracted the data. The 14-item Quality Assessment Tool for Observational Cohort and Cross-Sectional Studies ${ }^{17-19}$ was used to evaluate the quality of the studies. There are 14 questions in the scale, two researchers (HZ and WL) scored each item with Y (which indicates 'yes'), $\mathrm{N}$ (which means 'no'), CD (which means 'cannot determine') or NA (which means 'not applicable'). Study quality was rated according to the percentage of ' $\mathrm{Y}$ ' allocations out of the total score $(50 \%$ as good, $30 \%-50 \%$ as fair and below $30 \%$ as poor). This study was registered in the International Prospective Register of Systematic Reviews (CRD 42020171485).

\section{Psychological support policy for healthcare workers}

We searched the Chinese government website (www.gov. cn) for all national policies on the topic of healthcare starting from 1 January 2020 . We recorded policies relevant to the psychological support of healthcare workers. Document-issuing authorities included the State Council, the National Disease Control and Prevention Bureau, the State Council Joint Prevention and Control Team to Respond to the Novel Coronavirus Pneumonia and the National Health Commission.

\section{Statistical analysis}

$\mathrm{R}$ software V.3.5.1 was used to calculate the aggregate prevalence of individual psychological issues using the data extracted from the included studies. The 'metaprop' function in the 'meta' package was used to calculate the aggregate prevalence of each psychological issue. A cumulative meta-analysis was performed to show the changing trend of effect size over time: we sorted the included studies according to the date of research survey or publication, subsequently used the 'metacum' function in the meta package to present the cumulative forest plots. Heterogeneity was examined using $\mathrm{I}^{2}$ statistics. According to the Cochrane handbook, an $\mathrm{I}^{2}$ index ranging from $0 \%$ to $40 \%$ indicates that heterogeneity might not be important; 30\%-60\% may represent moderate heterogeneity; 50\%-90\% may represent substantial heterogeneity; and $75 \%-100 \%$ may represent considerable heterogeneity. In cases where heterogeneity among studies was regarded as low, a fixed-effect model was used to compute the pooled estimate of prevalence. Otherwise, a randomeffects model was used for the analysis. We also performed 


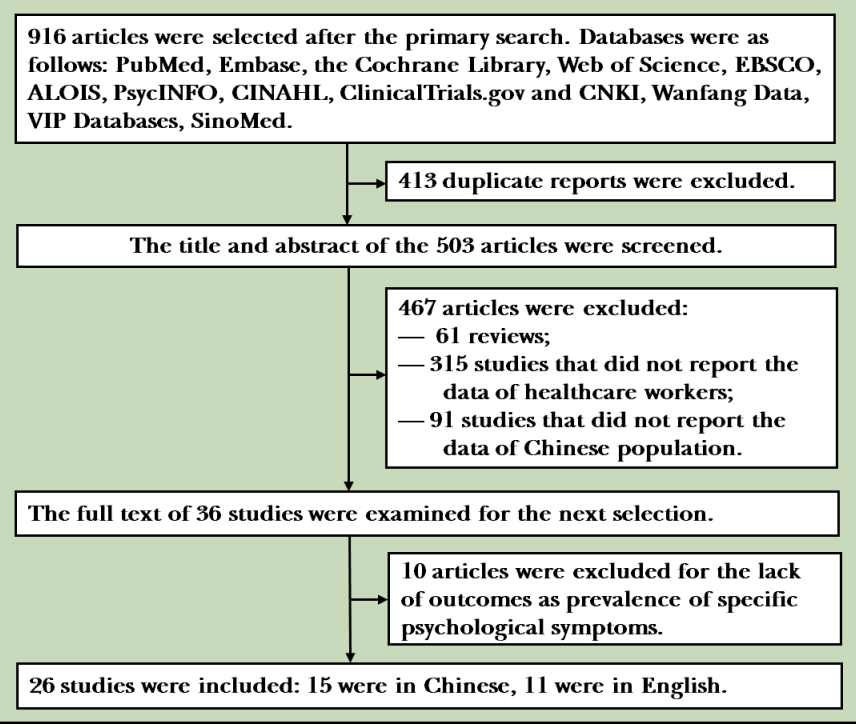

Figure 1 Flowchart of collecting and evaluating articles.

a sensitivity analysis in cases of moderate to high heterogeneity to identify the study that could account for more than $10 \%$ of heterogeneity. Publication bias was examined using Egger's test.

\section{RESULTS}

\section{Characteristics of included studies}

A flowchart outlining the literature screening process is presented in figure 1. A total of 26 articles were included in this review ${ }^{10-44}$ (table 1), with a total of 22062 medical workers surveyed. Fifteen articles were in Chinese, and 11 were in English. Eighteen studies $^{10} 2123$ 25-32 35-38 414244 reported the investigation time in the articles, and two studies' survey times were obtained from the authors. ${ }^{22}{ }^{24}$ For the remaining six studies $^{20} 3334394043$, admission time was used to estimate the approximate research time. The median survey time was from 31 January to 27 February, and the average duration of the survey was 2-14 days. The number of sampled populations in the studies ranged from 37 to 4369. Ten studies provided the information on the average age of the participants ${ }^{20} 2123-253039404244$; the mean age of the participants in these studies varied from 29.0 to 38.28 years old. Nineteen studies reported gender proportions $^{1021-2326-28303134-4244}$, and the ratio of men to the total population varied from $0 \%$ to $82.7 \%$. Overall, the included studies involved healthcare workers in 12 different provinces or municipalities. Four studies were nationwide surveys or had no location limitations 10 $263132^{26}$ five studies were conducted in Hubei province 252930364 ; and the other studies were conducted in Guangdong ${ }^{40}$, Beijing $^{20}{ }^{23}$, Tianjin $^{21}$, Henan $^{34}$, Hunan ${ }^{43}$, Anhui ${ }^{42}{ }^{44}$, Zhejiang $^{35}{ }^{38}$, Fujian ${ }^{28}$, Shanghai ${ }^{22} 27$, Sichuan ${ }^{33} 3739$ and Guizhou $^{24}$ (see table 1). The psychological issues surveyed and their corresponding assessment tools were anxiety symptoms (assessed with the Hamilton Anxiety Scale, the Self-rating Anxiety Scale, Generalised Anxiety
Disorder (GAD)-7 or GAD-2 and the Beck Anxiety Inventory), depression symptoms (assessed with the Hamilton Depression Scale, the Self-rating Depression Scale, the Patient Health Questionaire-9 or 2, Beck Depression Inventory-II and the Centre for Epidemiological Studies Depression Scale), sleep disturbance (assessed with the Insomnia Severity Index, the Impact of Events Scale-Revised and the Pittsburgh Sleep Quality Index) and stress-related problems (assessed with Perceived Stress Scale, Post-traumatic Stress Disorder Self-rating Scale, Maslach Burn-out Inventory and PTSD ChecklistCuvukuab Version) (see details in table 1).

\section{Quality of included studies}

As summarised in online supplemental table A1, using the Quality Assessment Tool for Observational Cohort and Cross-Sectional Studies, 7 studies were rated as good quality 22 26 28-30 41 44; 18 studies were rated as fair quality $^{102021} 23-252731-394243$ and 1 study was rated as poor quality. ${ }^{40}$

\section{Anxiety symptoms}

In total, 23 studies $^{10} 21-3537$ 39-44 investigated the anxiety level of medical workers $(n=21447)$. The results were summarised and showed that from 31 January to 27 February, the aggregate prevalence of anxiety symptoms among medical staff was $27.0 \%$ (95\% CI: $0.21 \%$ to $0.34 \%$, $\mathrm{I}^{2}=99.0 \%$ ). Sensitivity analysis revealed that $\mathrm{I}^{2}$ did not decrease significantly when any study was performed. Publication bias was not present, as confirmed by Egger's regression test (intercept $=-0.553, \mathrm{p}=0.58$ ), and cumulative meta-analysis found that a downward trend was observed over the time period (see figure 2). Between 31 January and 5 February, the detection rate of anxiety levels ranged from $37.7 \%$ to $56.3 \%$. An obvious decline in the detection rate was observed between 10 and 12 February, with a decrease from $37.2 \%$ to $32.0 \%$ from 13 and 27 February, and the rates of anxiety levelled off $(27.0 \%-30.8 \%)$.

\section{Depression symptoms}

In total, 18 studies ${ }^{10} 20-3234353842$ investigated the depression levels of Chinese medical workers during the COVID-19 pandemic under review $(\mathrm{n}=18818)$. Pooled results found that from 31 January to 27 February, the aggregate prevalence of depression among medical workers was $26.2 \%$ (95\% CI: $0.21 \%$ to $0.33 \%, \mathrm{I}^{2}=98.6 \%$ ). Sensitivity analysis revealed that $\mathrm{I}^{2}$ did not decrease significantly when any study was performed. Publication bias was not present, as confirmed by Egger's regression test (intercept $=-0.644, \mathrm{p}=0.52$ ), and cumulative metaanalysis also indicated a downward trend (figure 3). From 31 January to 5 February, the detection rate of depression ranged from $37.2 \%$ to $50.6 \%$. The detection rate of depressive symptoms decreased to $29.7 \%$ on 15 February. Then, until 27 February, the detection rate of depressive symptoms fluctuated between $26.2 \%$ and $29.2 \%$. 
Table 1 Characteristics of included studies

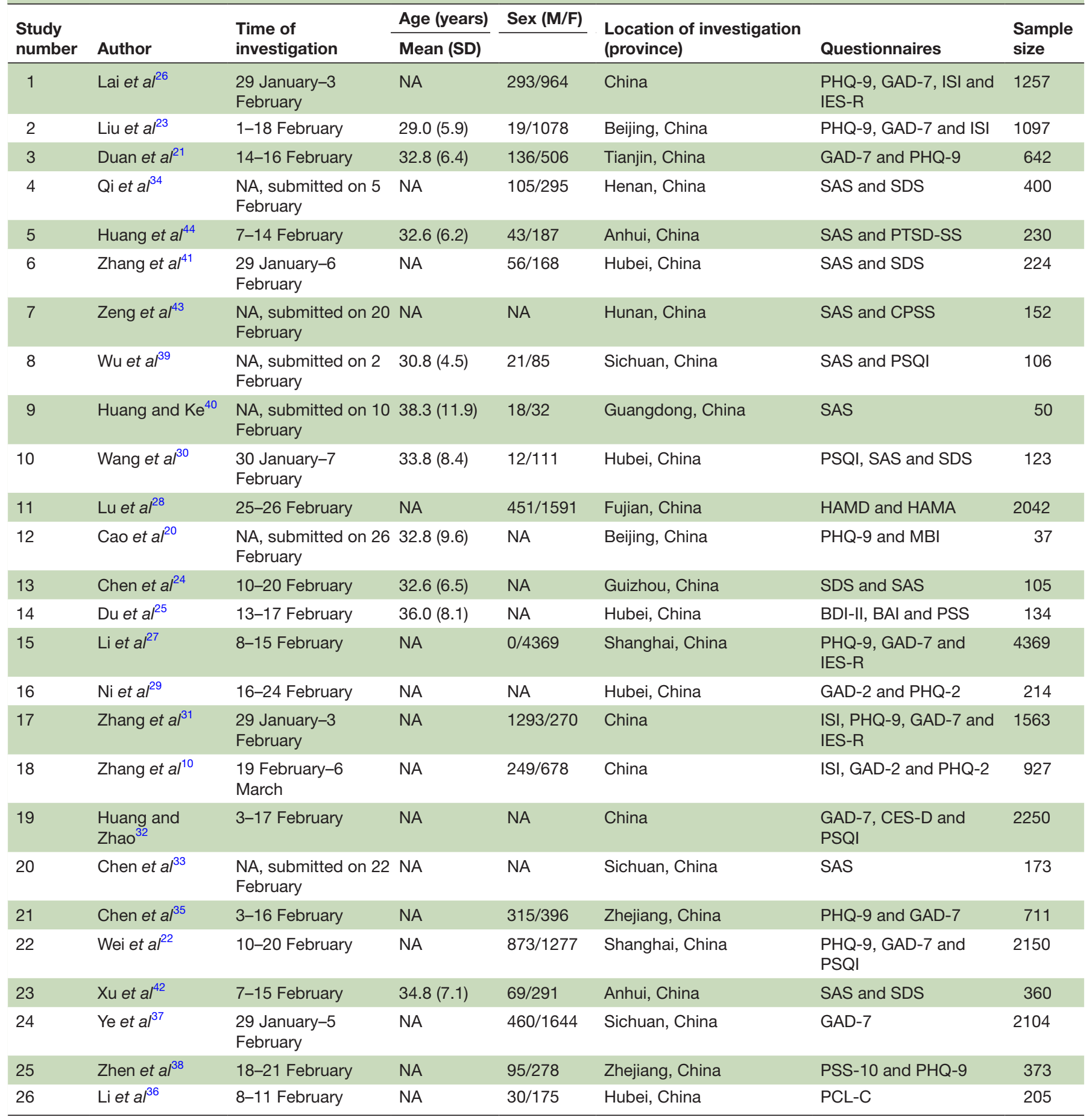

BAI, Beck Anxiety Inventory; BDI-II, Beck Depression Inventory-II; CES-D, Centre for Epidemiological Studies Depression Scale; CPSS, Chinese Perceived Stress Scale; GAD-2, Generalised Anxiety Disorder-2; GAD-7, Generalised Anxiety Disorder-7; HAMA, Hamilton Anxiety Scale; HAMD, Hamilton Depression Scale; IES-R, Impact of Events Scale-Revised; ISI, Insomnia Severity Index; MBI, Maslach Burn-out Inventory; NA, not applicable; PCL-C, PTSD Checklist-Civilian Version; PHQ-2, Patient Health Questionaire-2; PHQ-9, Patient Health Questionaire-9; PSQI, Pittsburgh Sleep Quality Index; PSS, Perceived Stress Scale; PTSD-SS, Post-Traumatic Stress Disorder Self Rating Scale; SAS, Self-rating Anxiety Scale; SDS, Self-rating Depression Scale; SQR-20, Self-reporting Questionnaires 20.

\section{Stress-related problems}

In total, eight studies ${ }^{23}$ 25-27 31364344 reported the prevalence of stress-related symptoms among medical staff from 13 January to 18 February $(n=9183)$. The aggregate prevalence of stress-related problems among medical workers during this period was $42.1 \%$ (95\% CI: $0.26 \%$ to $\left.0.60 \%, \mathrm{I}^{2}=99.5 \%\right)$. Sensitivity analysis revealed that the $\mathrm{I}^{2}$ statistic did not decrease significantly when interpreting any study. Publication bias was not conducted using Egger's regression test because of the small number of studies. The cumulative meta-analysis revealed a general downward trend (figure 4). In the early stages of the 
Study

Adding Lai J 01/31 ( $k=1$ Adding Zhang $C$ 01/31 $(\mathrm{k}=2)$ Adding Wu J 02/02 ( $\mathrm{k}=3$ ) Adding Ye J 02/02 ( $k=4)$

Adding Wang S 02/03 $(k=5)$

Adding Zhang Y 02/03 $(k=6)$

Adding Qi J 02/05 ( $k=7)$

Adding Huang $X 02 / 10(k=8)$

Adding Huang J 02/10 ( $k=9)$

Adding Chen Y 02/10 $(k=10)$

Adding Wei $L$ 02/10 ( $k=11)$

Adding $X u Y ~ 02 / 11(\mathrm{k}=12)$

Adding Li G 02/12 ( $\mathrm{k}=13)$

Adding Chen $Y 02 / 15(k=14)$

Adding Du J 02/15 ( $\mathrm{k}=15$ )

Adding Duan L 02/15 ( $\mathrm{k=16}$ )

Adding Liu X 02/18 ( $\mathrm{k}=17)$

Adding Zeng $M$ 02/20 ( $\mathrm{k}=18$ )

Adding Ni M 02/20 ( $\mathrm{k}=19)$

Adding Chen J 02/22 ( $k=20)$

Adding Wei L 02/24 ( $k=21)$

Adding Lu W 02/25 ( $\mathrm{k}=22$ )

Adding Zhang W 02/27 ( $\mathrm{k}=23$ )

Random effects model

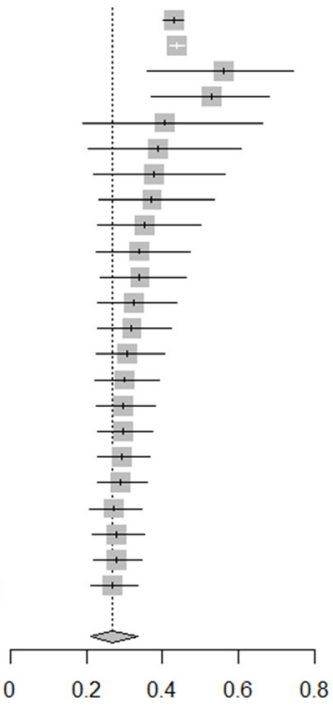

Proportion $\quad 95 \% \mathrm{Cl}$

Study

Proportion

$95 \% \mathrm{Cl}$

Figure 2 Cumulative meta-analysis of anxiety prevalence among Chinese healthcare workers.

pandemic, the prevalence was relatively high, ranging from $66.2 \%$ to $72.6 \%$, and on 12 February, the prevalence of stress-related problems ranged from $42.1 \%$ to $51.2 \%$.

\section{Sleep problems}

In total, eight studies ${ }^{10} 22232630-3239$ investigated the prevalence of sleep problems among medical workers $(\mathrm{n}=11758)$. The results revealed that from 31 January to 27 February, the aggregate prevalence of sleep problems among medical workers was $34.5 \%$ (95\% CI: $0.28 \%$ to $0.42 \%, \mathrm{I}^{2}=98.3 \%$ ). Sensitivity analysis revealed that $\mathrm{I}^{2} \mathrm{did}$ not decrease significantly when any study was performed. Publication bias was not conducted using Egger's regression test because of the small number of studies. The prevalence of sleep disturbance peaked around 2 January $(43.8 \%)$, followed by a slight downward trend over time, which levelled off between $34.1 \%$ and $34.9 \%$ after 18 January (figure 5).

Study

Adding Lai $\mathrm{J}(\mathrm{k}=1)$

Adding Zhang $\mathrm{C}(\mathrm{k}=2)$

Adding Wang $S(\mathrm{k}=3)$

Adding Qi J $(\mathrm{k}=4)$

Adding Chen $Y(k=5)$

Adding Huang $Y(k=6)$

Adding $X u Y(k=7)$

Adding Li $\mathrm{G}(\mathrm{k}=8)$

Adding Chen $Y(k=9)$

Adding Du J $(\mathrm{k}=10)$

Adding Duan $\mathrm{L}(\mathrm{k}=11)$

Adding Liu $X(k=12)$

Adding Zhen C $(\mathrm{k}=13)$

Adding Ni M ( $k=14)$

Adding Wei $\mathrm{L}(\mathrm{k}=15)$

Adding Lu W ( $\mathrm{k}=16)$

Adding Cao J ( $\mathrm{k}=17)$

Adding Zhang W ( $\mathrm{k}=18)$

Random effects model

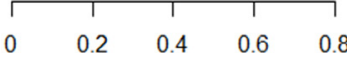

Figure 3 Cumulative meta-analysis of depression prevalence among Chinese healthcare workers.
$0.430-[0.402 ; 0.457]$

$0.439[0.421 ; 0.458]$

$0.563[0.360 ; 0.746]$

$0.529[0.372 ; 0.681]$

$0.407 \quad[0.192 ; 0.665]$

$0.388 \quad[0.207 ; 0.607]$

$0.377 \quad[0.220 ; 0.564]$

$0.372[0.233 ; 0.537]$

$0.355[0.230 ; 0.503]$

$0.339[0.226 ; 0.473]$

$0.341[0.238 ; 0.462]$

$0.326 \quad[0.230 ; 0.439]$

$0.320[0.231 ; 0.423]$

$0.308[0.226 ; 0.406]$

$0.300 \quad[0.223 ; 0.391]$

$0.298 \quad[0.225 ; 0.382]$

$0.297[0.228 ; 0.375]$

$0.293[0.229 ; 0.367]$

$0.289[0.228 ; 0.359]$

$0.272 \quad[0.209 ; 0.346]$

$0.279 \quad[0.217 ; 0.352]$

$0.278 \quad[0.219 ; 0.347]$

$0.270[0.212 ; 0.337]$

$0.270[0.212 ; 0.337]$

Adding Lai J ( $\mathrm{k}=1$ )

Adding Zhang $C(k=2)$

Adding $\mathrm{Li} \mathrm{C}(\mathrm{k}=3)$

Adding Huang J $(k=4)$

Adding Li G ( $\mathrm{k}=5)$

Adding Du J $(\mathrm{k}=6)$

Adding Liu X ( $\mathrm{k}=7$ )

Adding Zeng $M(k=8)$

Random effects model

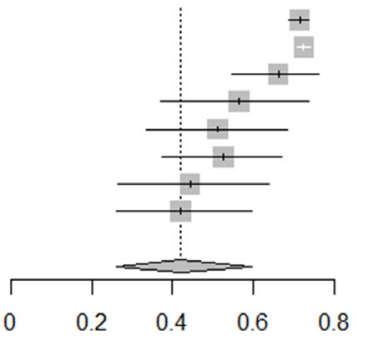

$0.715 \quad[0.690 ; 0.739]$ 0.726 [0.709; 0.742] $0.662[0.546 ; 0.762]$ $0.564[0.370 ; 0.740]$ $0.512[0.337 ; 0.685]$ $0.525[0.375 ; 0.671]$ $0.443 \quad[0.265 ; 0.638]$ $0.421[0.262 ; 0.598]$

$0.421[0.262 ; 0.598]$

Figure 4 Cumulative meta-analysis of stress-related problem prevalence among Chinese healthcare workers.

\section{National policies on psychological support for medical workers}

As shown in online supplemental table A2, the National Disease Control and Prevention Bureau launched the 'Principles for Emergency Psychological Crisis Intervention for the COVID-19 Pandemic', ${ }^{13}$ which emphasised the priority of psychological support and intervention for frontline medical staff. On 7 and 10 February, several policies were issued to improve the care of medical personnel in terms of security, work environment, family needs and psychological support. ${ }^{14} 45$ On 15 February, the National Health Commission launched a policy ${ }^{46}$ requiring psychiatric medical personnel to support Hubei Province, and specific medical service lines and special areas were set up in the existing psychological hotline and psychological assistance network. From 25 February to 18 March, several subsequent policies were released to further improve the care for medical workers and their families. ${ }^{47-49}$ Professional interventions such as psychological counselling and wholistic care were emphasised in these notices.

\section{DISCUSSION}

\section{Main findings}

This study reviewed papers surveying the psychological status of medical workers since the outbreak of the novel coronavirus epidemic in China. We found that from the early stage to the peak stage of the COVID-19 pandemic, medical workers had a high prevalence of psychological problems, including anxiety, depression, sleep problems and stress-related problems, which were several times higher than the equivalent prevalence of mental illness in the general population. ${ }^{50}$ The psychological problems of

$0.506[0.487 ; 0.524]$

$0.422[0.293 ; 0.563]$

$0.372[0.253 ; 0.508]$

$0.348 \quad[0.247 ; 0.464]$

$0.318 \quad[0.227 ; 0.426]$

0.327 [0.245; 0.422]

$0.297[0.215 ; 0.395]$

$0.297 \quad[0.222 ; 0.384]$

$0.275 \quad[0.203 ; 0.362]$

$0.280 \quad[0.212 ; 0.360]$

$0.288 \quad[0.223 ; 0.363]$

$0.290 \quad[0.229 ; 0.360]$

$0.282[0.225 ; 0.348]$

$0.292[0.235 ; 0.358]$

$0.278 \quad[0.220 ; 0.344]$

0.273 [0.218;0.337]

$0.262[0.208 ; 0.325]$

$0.262[0.208 ; 0.325]$

Study

Proportion $\quad 95 \% \mathrm{Cl}$

Adding Lai $\mathrm{J}(\mathrm{k}=1)$

Adding Zhang $\mathrm{C}(\mathrm{k}=2)$

Adding Wu $\mathrm{J}(\mathrm{k}=3)$

Adding Wang $S(k=4)$

Adding Huang $Y(k=5)$

Adding Liu $X(\mathrm{k}=6)$

Adding $\mathrm{Lu} \mathrm{L}(\mathrm{k}=7)$

Adding Wei $\mathrm{L}(\mathrm{k}=8)$

Adding Zhang W ( $k=9$ )

Random effects model

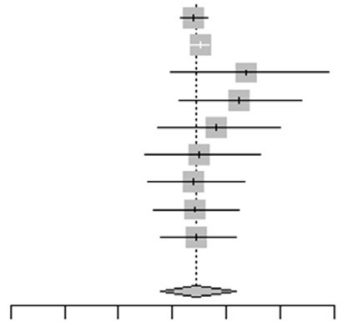

$0.340 \quad[0.314 ; 0.366]$

$0.351[0.334 ; 0.369]$

$0.438[0.296 ; 0.591]$

$0.423[0.314 ; 0.540]$

$0.381[0.274 ; 0.501]$

$0.349[0.250 ; 0.463]$

$0.339[0.254 ; 0.435]$

$0.341[0.266 ; 0.424]$

0.345 [0.278; 0.419]

$\begin{array}{lllllll}0 & 0.1 & 0.2 & 0.3 & 0.4 & 0.5 & 0.6\end{array}$

$0.345[0.278 ; 0.419]$

Figure 5 Cumulative meta-analysis of sleep problem

prevalence among Chinese healthcare workers. 
medical workers were particularly prominent in the early stages of the pandemic, followed by a slight downward trend and later a levelling off.

The occurrence of the pandemic has brought tremendous changes to people's lives and has also caused great psychological challenges ${ }^{51}$, especially for vulnerable populations. ${ }^{52}$ We observed that a significant proportion of healthcare workers dedicated to fighting COVID-19 are at-risk population, suffering from anxiety, depression, sleep problems and stressrelated problems throughout the entire survey period. This result was consistent with previous studies investigating the SARS pandemic, in which psychological problems were also found to be severe and frequent among medical staff. ${ }^{53}{ }^{54}$ We further found that psychological problems were especially robust in the early stages of other pandemics. This is understandable, given that medical workers face high levels of stress during the early stages of a pandemic owing to a rapid increase in workload, a shortage of supplies, uncertainty about the disease and the risk of infection. The numerous challenges are a significant and continuous source of stress, which can lead to a higher prevalence of acute stress disorders, anxiety, insomnia and overall psychological problems, especially among frontline healthcare workers. ${ }^{55}$ Studies have found that longer work hours and less social support are also related to depressive symptoms ${ }^{56}$ Furthermore, medical workers' sleep time and quality were also unavoidably affected by the challenging work environment and stressful mental state. ${ }^{57}$

Our study found that the prevalence of psychological problems among medical workers during the pandemic did not follow the upward growth curve of confirmed COVID-19 cases but instead showed a gentle decline. We speculated that this might be attributed to the implementation of comprehensive and timely measures during this period. As early as 27 January, the State Council declared the Principles for Emergency Psychological Crisis Intervention For the COVID-19 Pandemic ${ }^{13}$, which emphasised psychological interventions and support for medical staff. This indicated that policymakers paid close attention to the psychological problems of medical workers during the early stage of the pandemic. Moreover, 344 medical teams across the country were dispatched to Wuhan by $24 \mathrm{January}^{58}$, and several makeshift hospitals were built in a very short time, which undoubtedly eased the workload of local medical staff. Through the efforts of government departments and civil organisations, the difficult situation at the beginning of the pandemic was gradually eased. Medical protective equipment was fully supplied, and the order of patients visiting the hospital was set up. Additionally, further policies were introduced to solve various problems and needs that medical staff might encounter, such as the need to take care of elderly family members ${ }^{14}$, the problem of children's schooling and other family-related problems.
Furthermore, from 15 February onwards, specialised psychiatrists were added to the medical assistance teams to provide psychological support for workers at the epicentre of the pandemic. ${ }^{47}$ Moreover, online medical health services and psychological hotlines were also widely available during the pandemic ${ }^{59}$ and also offered help to those who were in adverse situations. Institutions and organisations also responded actively to the calls of national policies and provided psychological services for medical staff during the pandemic. For example, the Chinese Psychological Association launched the 'Anxin' ('relief' in English) campaign against the pandemic, which provided many psychological services such as professional lectures, self-assessments, online short-term psychological intervention programmes and adaptive online psychological counselling. ${ }^{60}$ The Shanghai Mental Health Centre cooperated with Shanghai People's Broadcasting Station and an online broadcasting platform to deliver psychological decompression lessons for medical staff on the radio. ${ }^{61}$ Although no studies quantify the impact of each policy or measure, their positive effect is evident from the changes in the psychological status of medical workers.

\section{Limitations}

This study has several implications. First, it was found that psychological issues peaked rapidly at the early stage of the pandemic, which suggests that psychological interventions for medical staff should be provided as early as possible. This is a call to reflect on the emergency response system of psychological interventions. First, it is undoubtedly necessary to ensure psychosocial preparedness by setting up specific mental health organisations and well-established emergency response systems for future pandemics. Various measures should be developed and integrated into the systems to help relieve the psychological burden of healthcare personnel, such as implementing self-coping strategies and psychosocial interventions and providing hospital support systems and occupational health policies. ${ }^{62}$ Online mental health services and social media have also proved to be effective tools and have played a crucial role in the battle against this disease. ${ }^{59}$ In the future, by improving the construction of policies and systems, policies can be implemented, and interventions can reduce the stress response and negative experiences of medical workers during health emergencies. ${ }^{63}$ Second, we found that medical workers still exhibited a high level of psychological distress during the later period of the pandemic; therefore, greater attention should be paid to those enduring psychological effects. The prevalence of post-traumatic stress symptoms among frontline healthcare workers reached nearly $10 \%$ even several years after the SARS outbreak. ${ }^{64}$ This indicated that it was vital to perform further follow-up visits and to provide support for medical staff after the acute phase of COVID-19. 
It should be mentioned that our study did not reach a causal association or statistical relationship between policies and psychological status among healthcare workers. The time interval from the launch of policies to their specific implementation is unknown to us. Further, the implementation status of policies in different regions may also be different. Moreover, during the pandemic, other influencing factors would also have an impact on medical workers' psychological condition, such as support from civil society, the development phase of the pandemic, their personal support structures, and the differences in individuals' basic psychological characteristics. Therefore, the impact of policies on psychological status must be interpreted cautiously.

The following limitations should be considered when interpreting the findings of this study. First, the current number of the studies and the total number of samples included in the studies were relatively small, and the participants in most studies were enrolled by convenience sampling rather than probabilistic sampling, leading to a limited representativeness of the sample population. However, owing to the urgent and special situation at the beginning of the pandemic, it was not feasible to conduct large-scale epidemiological investigations on medical staff's psychological status at that time; thus, our meta-analysis may provide alternative information on that question. Second, there were significant heterogeneities between studies, which may arise from inter-regional differences in the sampled location or methodological differences in the assessment tools applied. Owing to the limited number of the studies, it is not feasible to conduct further subgroup analyses to analyse the source of heterogeneity. Several studies had a wide survey time span; thus, there were some errors in the method of selecting the median time of the survey to represent the incidence within the study period. Therefore, more largescale and longitudinal studies are needed to reach firmer conclusions.

\section{Implications}

During the pandemic, Chinese medical workers exhibited significant psychological problems, with the peak occurring in the early stage of the pandemic and gradually showing a downward trend. However, there was still a high prevalence of psychological problems during the middle and later stages of the pandemic. Implementation of relevant supportive policies may help reduce psychological problems, especially in preventing the incidence of delayed mental health problems among this population.

\section{Author affiliations}

${ }^{1}$ Shanghai Key Laboratory of Psychotic Disorders, Shanghai Mental Health Center,

Shanghai Jiao Tong University School of Medicine, Shanghai, China

${ }^{2}$ Clinical Research Center, Shanghai Mental Health Center, Shanghai Jiao Tong

University School of Medicine, Shanghai, China

${ }^{3}$ Department of Medical Psychology, Shanghai General Hospital, Shanghai Jiao Tong University, Shanghai, China
${ }^{4}$ Institute of Psychology and Behavioral Science, Shanghai Jiao Tong University, Shanghai, China

${ }^{5}$ Shanghai Mental Health Center, Shanghai Jiao Tong University School of Medicine, Shanghai, China

\section{Twitter Yifeng Xu @NO}

Contributors $\mathrm{HL}$ and $\mathrm{CL}$ designed the study. $\mathrm{HZ}$ and $\mathrm{WL}$ drafted the manuscript. WL and $H L$ were responsible for drafting the research searching strategy and data extraction. JL, $\mathrm{CZ}$ and $\mathrm{YZ}$ conducted the searching and screening of studies. $\mathrm{HL}, \mathrm{ZZ}$, YX, CL, JW and HW made critical revisions. All authors read and approved the final version of the manuscript.

Funding This work was supported by Shanghai Clinical Research Center for Mental Health (19MC1911100), Shanghai Jiao Tong University Special Grant for the Prevention and Control of Novel Coronavirus (2020RK61).

Competing interests None declared.

Patient consent for publication Not required.

Provenance and peer review Not commissioned; externally peer reviewed.

Data availability statement No additional data are available.

Supplemental material This content has been supplied by the author(s). It has not been vetted by BMJ Publishing Group Limited (BMJ) and may not have been peer-reviewed. Any opinions or recommendations discussed are solely those of the author(s) and are not endorsed by BMJ. BMJ disclaims all liability and responsibility arising from any reliance placed on the content. Where the content includes any translated material, BMJ does not warrant the accuracy and reliability of the translations (including but not limited to local regulations, clinical guidelines, terminology, drug names and drug dosages), and is not responsible for any error and/or omissions arising from translation and adaptation or otherwise.

Open access This is an open access article distributed in accordance with the Creative Commons Attribution Non Commercial (CC BY-NC 4.0) license, which permits others to distribute, remix, adapt, build upon this work non-commercially, and license their derivative works on different terms, provided the original work is properly cited, appropriate credit is given, any changes made indicated, and the use is non-commercial. See: http://creativecommons.org/licenses/by-nc/4.0/.

ORCID iDs

Huijuan Zhang http://orcid.org/0000-0002-3791-7133

Yifeng Xu http://orcid.org/0000-0002-0403-1141

\section{REFERENCES}

1 Wang C, Horby PW, Hayden FG, et al. A novel coronavirus outbreak of global health concern. Lancet 2020;395:470-3.

2 Carlos WG, Dela Cruz CS, Cao B, et al. Novel Wuhan (2019-nCoV) coronavirus. Am J Respir Crit Care Med 2020;201:P7-8.

3 World Health Organization. Who coronavirus disease (COVID-19) Dashboard, 2020. Available: https://covid19.who.int/ [Accessed 5 Sep 2020].

4 The National Health Commission of China. Updates on the novel coronavirus outbreak up to may 8, 2020, 2020. Available: http://www. nhc.gov.cn/xcs/yqtb/202005/2e48fde64f4a4f52b71724050222fbfb. shtml [Accessed 8 May 2020].

5 Wu Z, McGoogan JM. Characteristics of and Important Lessons From the Coronavirus Disease 2019 (COVID-19) Outbreak in China: Summary of a Report of 72314 Cases From the Chinese Center for Disease Control and Prevention. JAMA 2020;323:1239-42.

6 Pan A, Liu L, Wang C, et al. Association of public health interventions with the epidemiology of the COVID-19 outbreak in Wuhan, China. JAMA 2020;323:1915-23.

7 Kang L, Li Y, Hu S, et al. The mental health of medical workers in Wuhan, China dealing with the 2019 novel coronavirus. Lancet Psychiatry 2020;7:e14.

8 Wu W, Zhang Y, Wang P, et al. Psychological stress of medical staffs during outbreak of COVID-19 and adjustment strategy. J Med Virol 2020;92:1962-70.

9 Cai H, Tu B, Ma J, et al. Psychological Impact and Coping Strategies of Frontline Medical Staff in Hunan Between January and March 2020 During the Outbreak of Coronavirus Disease 2019 (COVID-19) in Hubei, China. Med Sci Monit 2020;26:e924171.

10 Zhang W-rui, Wang K, Yin L, et al. Mental health and psychosocial problems of medical health workers during the COVID-19 epidemic in China. Psychother Psychosom 2020;89:242-50. 
11 Li W, Zhang CD, Luo JJ. Psychological status among different populations during COVID-19 epidemic: a systematic review and meta-analysis. J Tongji Univ 2020;41:147-54.

12 Wang Y, Zhao X, Feng Q, et al. Psychological assistance during the coronavirus disease 2019 outbreak in China. $J$ Health Psychol 2020;25:733-7.

13 The National Disease Control and Prevention Bureau. Principles for emergency psychological crisis intervention for COVID-19 pneumonia epidemic [EB/OL], 2020. Available: http://www.nhc.gov. cn/jkj/s3577/202001/6adc08b966594253b2b791be5c3b9467.shtml [Accessed 27 Jan 2020].

14 The State Council. Notice on earnestly ensure the support of frontline medical personnel and their families [EB/OL], 2020. Available: http:// www.nhc.gov.cn/renshi/s7746/202002/d919a2a8313144b399065e6e b825e9b1.shtml [Accessed 7 Feb 2020].

15 Maunder RG, Lancee WJ, Balderson KE, et al. LongTerm psychological and occupational effects of providing Hospital healthcare during SARS outbreak. Emerg Infect Dis 2006;12:1924-32.

16 McAlonan GM, Lee AM, Cheung V, et al. Immediate and sustained psychological impact of an emerging infectious disease outbreak on health care workers. Can J Psychiatry 2007;52:241-7.

17 National Institute of Health: US Department of Health and Human Services. Quality assessment tool for observational cohort and crosssectional studies, 2017.

18 Aasa U, Svartholm I, Andersson F, et al. Injuries among weightlifters and powerlifters: a systematic review. Br J Sports Med 2017;51:211-9.

19 Low ZX, Yeo KA, Sharma VK, et al. Prevalence of burnout in medical and surgical residents: a meta-analysis. Int J Environ Res Public Health 2019;16:1479.

$20 \mathrm{Cao} \mathrm{J}$, Wei J, Zhu H, et al. A study of basic needs and psychological wellbeing of medical workers in the fever clinic of a tertiary General Hospital in Beijing during the COVID-19 outbreak. Psychother Psychosom 2020;89:252-4.

21 Duan L, Guo Y, Sun J. Mental health status of officers and soldiers in a military Hospital amid the epidemic of COVID-19. Medical Journal of the Chinese People's Armed Police Force 2020;31:191-4.

22 Wei L, Shi L, Cao J. Psychological status of primary care workers during the COVID-19 epidemic. J Tongji Univ 2020:1-6.

23 Liu X, Cheng Y, Wang M. Psychological state of nursing staff in a large scale of General Hospital during COVID-19 epidemic. Chinese Journal of Nosocomiology 2020;30.

24 Chen Y, Zhou H, Zhou Y, et al. Prevalence of self-reported depression and anxiety among pediatric medical staff members during the COVID-19 outbreak in Guiyang, China. Psychiatry Res 2020;288:113005.

25 Du J, Dong L, Wang T, et al. Psychological symptoms among frontline healthcare workers during COVID-19 outbreak in Wuhan. Gen Hosp Psychiatry 2020;67:144-5.

26 Lai J, Ma S, Wang Y, et al. Factors associated with mental health outcomes among health care workers exposed to coronavirus disease 2019. JAMA Netw Open 2020;3:e203976.

$27 \mathrm{Li} \mathrm{G}$, Miao J, Wang H, et al. Psychological impact on women health workers involved in COVID-19 outbreak in Wuhan: a cross-sectional study. J Neurol Neurosurg Psychiatry 2020;91:895-7.

28 Lu W, Wang H, Lin Y, et al. Psychological status of medical workforce during the COVID-19 pandemic: a cross-sectional study. Psychiatry Res 2020;288:112936.

29 Ni MY, Yang L, Leung CMC, et al. Mental health, risk factors, and social media use during the COVID-19 epidemic and cordon sanitaire among the community and health professionals in Wuhan, China: cross-sectional survey. JMIR Ment Health 2020;7:e19009.

30 Wang S, Xie L, Xu Y, et al. Sleep disturbances among medical workers during the outbreak of COVID-2019. Occup Med 2020;70:364-9.

31 Zhang C, Yang L, Liu S, et al. Survey of insomnia and related social psychological factors among medical staff involved in the 2019 novel coronavirus disease outbreak. Front Psychiatry 2020;11:306.

32 Huang Y, Zhao N. Chinese mental health burden during the COVID-19 pandemic. Asian J Psychiatr 2020;51:102052.

33 Chen J, Min M, Yin X. Investigation and analysis of psychological anxiety of medical staff in Qingbaijiang district of Chengdu City during novel coronavirus pneumonia pandemic. Shandong Medical Journal 2020;60:70-2.

34 Qi J, Liu L, Li W. Investigation and analysis of clinical nurses' psychological status in face of COVID-19. Chin Gen Pract Nurs 2020;18:703-4.

35 Chen Y, Sun J, Hu J. Analysis of mental health status of residents during COVID-19 epidemic. Clinical Education of General Practice 2020;18:237-9.
$36 \mathrm{Li} \mathrm{C}, \mathrm{Mi} \mathrm{Y}$, Chu J. Investigation and analysis of post-traumatic stress disorder in first-line nursing staff of COVID-19. Journal of Nurses Training 2020;35:615-8.

37 Ye J, Xia Z, Xiao A. Investigation on nurses' anxiety status and influencing factors during the outbreak of COVID-19. Sichuan Mental Health 2020;33:11-14.

38 Zhen C, Shen F, Tian G. Investigation on the stress level and depression of medical staff during COVID-19 pandemic. Zhejiang Medicine 2020;42:406-7.

39 Wu J, Song X, Chen F. Investigation on sleep quality of first-line nurses in fighting against novel coronavirus pneumonia and its influencing factors. Chin Nurs Res 2020;34:558-62.

40 Huang $X$, Ke P. The effect of standardized training on the anxiety level of staff in the sterile supply center of COVID-19. Chinese General Practice Nursing 2020;18:548-50.

41 Zhang Y, Zhang X, Peng J. A survey on mental health of medical staff fighting 2019 novel coronavirus diseases in Wuhan. J Trop Med 2020;20.

$42 \mathrm{Xu}$ Y, Zhao M, Tang X. Correlation study between mental health and coping style of medical staff during COVID-19 epidemic. Anhui Medical Journal 2020:1-4.

43 Zeng M, Li X, Ou L. Effect of EFT on psychological pressure of firstline nurses against epidemic. Shanghai Journal of Acupuncture and Moxibustion 2020;39.

44 Huang JZ, Han MF, Luo TD. Mental health survey of 230 medical staff in a tertiary infectious disease Hospital for COVID-19. Chinese Journal of Industrial Hygiene and Occupational Diseases 2020;38:E001.

45 The State Council. Several measures to improve the working conditions of frontline medical staff and to care for their physical and mental health [EB/OL], 2020. Available: http://www.gov.cn/ zhengce/content/2020-02/11/content_5477399.htm [Accessed 10 Feb 2020].

46 The National Health Commission. Notice on the implementation of several measures to improve the working conditions of frontline medical staff and to care for their physical and mental health [EB/ OL], 2020. Available: http://www.nhc.gov.cn/renshi/s3577/202002/ 85896fabe90747cba8b79beb4c57f202.shtml [Accessed 15 Feb 2020].

47 The State Council. Notice on the comprehensive implementation of several measures to further protect and care for medical staff. [EB/ OL], 2020. Available: http://www.gov.cn/zhengce/content/2020-02/ 23/content_5482345.htm [Accessed 23 Feb 2020].

48 The National Disease Control and Prevention Bureau. Notice on strengthening psychological assistance and social work services in response to COVID-19 epidemic[EB/OL], 2020. Available: http:// www.nhc.gov.cn/jkj/s3577/202003/a9b0bcb3bb7445298c480c50 03c51d6d.shtml [Accessed 5 Mar 2020].

49 The National Disease Control and Prevention Bureau. Notice on the issuance of the work plan for psychological support during the outbreak of novel coronary pneumonia. [EB/OL], 2020. Available: http://www.nhc.gov.cn/jkj/s3577/202003/0beb22634f8a4a48aecf 405c289fc25e.shtml [Accessed 18 Mar 2020].

50 Huang $\mathrm{Y}$, Wang $\mathrm{Y}$, Wang $\mathrm{H}$, et al. Prevalence of mental disorders in China: a cross-sectional epidemiological study. Lancet Psychiatry 2019;6:211-24.

51 Qiu J, Shen B, Zhao M, et al. A nationwide survey of psychological distress among Chinese people in the COVID-19 epidemic: implications and policy recommendations. Gen Psychiatr 2020;33:e100213.

52 Yao H, Chen J-H, Xu Y-F. Patients with mental health disorders in the COVID-19 epidemic. Lancet Psychiatry 2020;7:e21.

53 Chua SE, Cheung V, Cheung C, et al. Psychological effects of the SARS outbreak in Hong Kong on high-risk health care workers. Can $J$ Psychiatry 2004;49:391-3.

54 Chen C-S, Wu H-Y, Yang P, et al. Psychological distress of nurses in Taiwan who worked during the outbreak of SARS. Psychiatr Serv 2005;56:76-9.

55 Que J, Shi L, Deng J, et al. Psychological impact of the COVID-19 pandemic on healthcare workers: a cross-sectional study in China. Gen Psychiatr 2020;33:e100259.

56 Liu Y, Zhang J, Hennessy DA, et al. Psychological strains, depressive symptoms, and suicidal ideation among medical and non-medical staff in urban China. J Affect Disord 2019;245:22-7.

57 Zhuo K, Gao C, Wang X, et al. Stress and sleep: a survey based on wearable sleep trackers among medical and nursing staff in Wuhan during the COVID-19 pandemic. Gen Psychiatr 2020;33:e100260.

58 Baidu Baike. National medical assisstant team to Hubei. Available: https://baike.baidu.com/item/

59 Liu S, Yang L, Zhang C, et al. Online mental health services in China during the COVID-19 outbreak. Lancet Psychiatry 2020;7:e17-18. 
60 The Chinese Psychological Association. "Relief" action against the epidemic-psychological assistance and crisis intervention to combat the COVID-19 epidemic, 2020. Available: https://www.cpsbeijing. $\mathrm{org} / \mathrm{cms} /$ show.action?code=publish_402880766305a05d016305ff c2710078\&siteid $=100000 \&$ newsid $=e b 5$ ea 1131 e064055972be41e 296a0f3d\&channelid $=0000000036$

61 Shanghai Mental Health Center. Decompression lecture series on medical staff: guardian angel, 2020. Available: https://mp.weixin.qq. com/s/QynGlbUpi3e6q7xg3KcKsw [Accessed 12 Feb 2020].
62 Cabarkapa S, Nadjidai SE, Murgier J, et al. The psychological impact of COVID-19 and other viral epidemics on frontline healthcare workers and ways to address it: a rapid systematic review. Brain Behav Immun Health 2020;8:100144.

63 Qin B, Sun Y, He Y. Psychological crisis intervention measures for major public health emergencies abroad and their enlightenment. $J$ Nanjing Med Univ 2020;20:116-22.

64 Wu P, Fang Y, Guan Z, et al. The psychological impact of the SARS epidemic on hospital employees in China: exposure, risk perception, and altruistic acceptance of risk. Can J Psychiatry 2009;54:302-11.

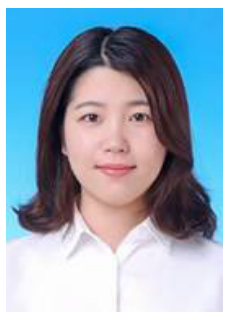

Huijuan Zhang obtained a doctor's degree in Psychiatry from Shanghai jiao Tong University, Shanghai, China, in 2019. At present, she is a resident doctor at the Department of Psychiatry in Shanghai Mental Health Center, Shanghai, China. Her main research interests include evidence-based medicine and cognitive function of the psychosis. 